PHYSICAL REVIEW D 93, 123014 (2016)

\title{
Constraining the Nordtvedt parameter with the BepiColombo Radioscience experiment
}

\author{
Fabrizio De Marchi, ${ }^{1,}$ Giacomo Tommei, ${ }^{2}$ Andrea Milani, ${ }^{2}$ and Giulia Schettino ${ }^{2}$ \\ ${ }^{1}$ Department of Mechanical and Aerospace Engineering, Sapienza University of Rome, \\ Via Eudossiana, 18, 00184 Rome, Italy \\ ${ }^{2}$ Department of Mathematics, University of Pisa, Largo Bruno Pontecorvo 6, 56127 Pisa, Italy
}

(Received 27 May 2016; published 22 June 2016)

\begin{abstract}
BepiColombo is a joint ESA/JAXA mission to Mercury with challenging objectives regarding geophysics, geodesy, and fundamental physics. The Mercury Orbiter Radioscience Experiment (MORE) is one of the on-board experiments, including three different but linked experiments: gravimetry, rotation, and relativity. The aim of the relativity experiment is the measurement of the post-Newtonian parameters. Thanks to accurate tracking between Earth and spacecraft, the results are expected to be very precise. However, the outcomes of the experiment strictly depend on our "knowledge" about solar system: ephemerides; number of bodies (planets, satellites, and asteroids); and their masses. In this paper we describe a semianalytic model used to perform a covariance analysis to quantify the effects on the relativity experiment, due to the uncertainties of Solar System bodies' parameters. In particular, our attention is focused on the Nordtvedt parameter $\eta$ used to parametrize the strong equivalence principle violation. After our analysis we estimated $\sigma[\eta] \lesssim 4.5 \times 10^{-5}$, which is about 1 order of magnitude larger than the "ideal" case where masses of planets and asteroids have no errors. The current value, obtained from ground-based experiments and lunar laser ranging measurements, is $\sigma[\eta] \approx 4.4 \times 10^{-4}$. Therefore, we conclude that, even in the presence of uncertainties on Solar System parameters, the measurement of $\eta$ by MORE can improve the current precision of about 1 order of magnitude.
\end{abstract}

DOI: 10.1103/PhysRevD.93.123014

\section{INTRODUCTION}

BepiColombo (BC) is a joint ESA/JAXA mission aimed at the exploration of Mercury. The mission is composed of two spacecrafts, the ESA Mercury Planetary Orbiter (MPO) and the JAXA Mercury Magnetospheric Orbiter (MMO), to be put in orbit around Mercury: the launch is scheduled for April 2018 and the orbit insertion for December 2024. The nominal duration of the whole mission is 1 year, with a possible extension to 2 years.

The Mercury Orbiter Radioscience Experiment (MORE) is one of the on-board experiments whose goals are

(a) to determine the gravity field of Mercury and its rotation state (gravimetry and rotation experiments);

(b) to study possible violations of the general relativity (GR) theory of gravitation (relativity experiment);

(c) to provide the spacecraft position for geodesy experiments;

(d) to contribute to planetary ephemerides improvement.

Such precise experiments are possible thanks to a multifrequency radio link (in $X$ and $K_{a}$ bands), allowing us to eliminate the uncertainty in the refraction index due to plasma content along the radio waves' path [1]. The MORE experiment provides the necessary $K_{a}$-band transponder and the system to compare the delays in a five-way link,

*Corresponding author. fabrizio.demarchi@uniroma1.it in combination with instruments installed at the ground stations.

Orders of magnitude for the accuracy which can be achieved in this way are 3 micron/s in range rate (two-way, at $1000 \mathrm{~s}$ of integration time) and $30 \mathrm{~cm}$ in range (two-way, at $300 \mathrm{~s}$ of integration time): the relative accuracy in range is better than $10^{-12}$. This implies that the signal to noise ratio $(\mathrm{S} / \mathrm{N})$ of all the relativistic effects (both in the dynamics and in the observation equations) is very large, in particular for the range measurements. By using the nonlinear least-squares method, range and range-rate data will be fitted to an accurate theoretical model to estimate the physical parameters relative to MORE as well as their uncertainties.

One of the most ambitious purposes of the mission is to attempt to constrain the Nordtvedt parameter $\eta$ describing violations of the strong equivalence principle (SEP). The equivalence principle (EP) states the equality between inertial and gravitational mass. As a consequence, there is the universality of the free fall that allows the geometrical description of gravity in GR. So far, it has not been experimentally disproved. In its weak form, the EP states that strong and electroweak interactions do not influence the falling of a body, with a negligible self-gravity, in an external gravity field. This is called the weak equivalence principle (WEP). The strong form extends the validity of the EP to bodies with measurable self-gravitational energy, 
like stars or planets. The inequality between inertial mass $m_{i}^{I}$ and gravitational mass $m_{i}^{G}$ of a body can be parametrized as [2-4]

$$
m_{i}^{G}=m_{i}^{I}\left(1+\delta_{i}+\eta \Omega_{i}\right),
$$

where $\Omega_{i}$ is the ratio between self-gravitational and rest energy of the body.

The validity of the WEP corresponds to $\delta_{i}=0$ and $\Omega_{i}=0$. On the other hand, when $\Omega_{i} \neq 0$, if both $\delta_{i}$ and $\eta$ are zero, then the SEP is valid. The values of $\Omega_{i}$ for the Sun, Earth, and Moon are, respectively, $\Omega_{s}=-3.52 \times 10^{-6}$, $\Omega_{e}=-4.64 \times 10^{-10}$, and $\Omega_{m}=-1.88 \times 10^{-11}$ [5].

Since Newton, EP has been tested several times by laboratory experiments, mainly by torsion balances, but laboratory objects have a very small self-gravity $\left(\Omega_{i} \lesssim 10^{-26}\right)$; therefore no information about SEP can arise from ground-based experiments only. Hence, an EP test is in practice a WEP test.

The first test of the EP involving celestial bodies (Earth, Moon, and Sun) has been proposed by Nordtvedt [6]: in case of EP violation the different rate of free falling of Earth and Moon toward the Sun generates a signal in the Earth-Moon range. This signal carries information about both WEP and SEP, since Earth and Moon have different gravitational self-energies but also compositions. Regarding the SEP violation, the amplitude of the signal at Earth-Moon distance is proportional to $\left(\Omega_{e}-\Omega_{m}\right)$, at the Moon's synodic frequency, and it has been estimated to be $\approx 13 \eta$ meters (the so-called Nordtvedt effect). In order to separate weak and strong EP, laboratory results involving test bodies with chemical compositions similar to those of Earth and Moon have been used. The precision achieved on WEP measurements is currently $\sigma\left[\delta a_{\mathrm{WEP}} / a\right]=1.4 \times 10^{-13}$ [7], but this result is expected to be improved by 2 orders of magnitude thanks to the recently launched ESA mission MICROSCOPE [8]. The Earth-Moon distance has been measured with increasing precision in the last 46 years by lunar laser ranging (LLR) [9] and the precision on Earth-Moon relative differential accelerations is currently $\sigma\left[\delta a_{e m} / a_{\odot}\right]=1.3 \times 10^{-13}[5]$. This result is comparable to that achieved by ground experiments.

In order to estimate $\eta$, it is necessary to consider both experiments, and the resulting root mean square (RMS) is $\sigma[\eta]=4.4 \times 10^{-4}[5,7,10]$. SEP violation can be tested also by planetary ranging, i.e., by radio-tracking between Earth and an object orbiting around the Sun (a spacecraft or another planet). The advantages with respect to the Earth-Moon tests are (i) the signal is proportional to $\Omega_{s}$ which is 4 orders of magnitude larger than $\Omega_{e}-\Omega_{m}$ and (ii) the baseline is in general larger [11].

A test for the SEP violation by ranging measurements between Earth and $L_{1}$ or $L_{2}$ Earth-Sun Lagrangian points has been recently proposed in [4], while the same experiment for Earth-Mars or Earth-Phobos ranging has been described in [10-12]. Thanks to precise Earth-Mercury range measurements, the $\mathrm{BC}$ mission will provide data to estimate with good accuracy $\eta$ and other post-Newtonian (PN) parameters $[2,13]$. Regarding the EP, the expected precision for relative Earth-Mercury acceleration is $\sigma\left[\delta a_{\text {earth-merc. }} / a_{\odot}\right] \approx 10^{-11}$. It is 2 orders of magnitude worse than the precision achieved by laboratory tests; therefore the $\mathrm{EP}$ violation test of $\mathrm{BC}$ is in practice a SEP test. Moreover, the SEP violation signal is proportional to $\Omega_{s}$; therefore the parameter $\eta$ can be estimated with high precision.

The purpose of this work is to quantify $\sigma[\eta]$ in the presence of systematic effects due to planets and asteroids. To do this, we will use both a numerical approach (by using the software ORBIT14, described in Sec. II) and an analytical one. This latter approach is necessary to take into account the effects of the experimental uncertainties of gravitational parameters $\left(\mu_{i}=G m_{i}^{G}\right.$, hereafter GPs $)$ of planets and asteroids.

This paper has the following structure. In Sec. II we are going to give a brief description of the mathematical models and of the structure of the simulation software used and we will present the result of a nominal simulation of the relativity experiment. Section III is devoted to the detailed description of the experiment to estimate $\eta$ and of the analytical method followed. Finally, in Sec. IV we will draw some conclusions.

\section{MATHEMATICAL MODELS AND SOFTWARE STRUCTURE}

In this section we briefly describe the mathematical models that are the basis of the software ORBIT14 built to process the data of MORE and used as simulation software to infer some results about the estimation of parameters. ORBIT14 has been developed in the last six years by the Celestial Mechanics Group of the University of Pisa and it has the capability to simulate the relativity experiment, the gravimetry and rotation experiment [14] of the BC mission, and also the radioscience experiment of the NASA mission JUNO [15].

Concerning the relativity experiment, we need to solve an orbit determination problem with a full relativistic model (also for the observable computations, see [16]), including the terms expressing the violations of general relativity with the PN parameters, such as $\gamma, \beta, \eta, \alpha_{1}, \alpha_{2}$.

The equations of motion of Mercury and of the Earth-Moon barycenter (EMB) have been implemented using the parametric post-Newtonian approach: they are linearized with respect to the small parameters $v_{i}^{2} / c^{2}$ and $G m_{i} /\left(c^{2} r_{i k}\right)$, where $v_{i}$ is the barycentric velocity for each of the bodies of mass $m_{i}$, the speed of light $c$, and a mutual distance $r_{i k}$, appearing in the metric of the curved spacetime and hence in the equations for geodesic motion. This can be done by adding to the Lagrangian of the N-body 
problem some corrective terms of PN order 1 in the small parameters (as described in [13]).

Here we are interested in the model for the violation of SEP. We can consider that there are for each body $i$ two quantities $\mu_{i}=G m_{i}^{G}$ and $\mu_{i}^{I}=G m_{i}^{I}$, one in the gravitational potential (including the relativistic part) and the other in the kinetic energy, not considering the relativistic correction for the masses. If there is a violation of the SEP involving body $i$, with a fraction $\Omega_{i}$ of its mass due to gravitational self-energy, then from Eq. (1) and neglecting WEP,

$$
\mu_{i}=\mu_{i}^{I}\left(1+\eta \Omega_{i}\right) \Leftrightarrow \mu_{i}^{I}=\mu_{i}\left(1-\eta \Omega_{i}\right)+\mathcal{O}\left(\eta^{2}\right) .
$$

Neglecting $\mathcal{O}\left(\eta^{2}\right)$ terms, this is expressed by a Lagrangian term $\eta L_{\eta}$, with an effect on body $i$ :

$L_{\eta}=-\frac{1}{2} \sum_{i} \Omega_{i} \mu_{i} v_{i}^{2} \Rightarrow \frac{d^{2} \mathbf{r}_{i}}{d t^{2}}=\left.\left(1+\eta \Omega_{i}\right) \frac{d^{2} \mathbf{r}_{i}}{d t^{2}}\right|_{\eta=0}$.

The largest effect of $\eta$ is a change in the center of mass integral (where dots stand for terms of order $2 \mathrm{PN}$ and higher, including the neglected terms in the masses)

$$
\mathbf{P}=\sum_{j} \frac{\partial L}{\partial \mathbf{v}_{j}}=\sum_{j}\left(1-\eta \Omega_{i}\right) \mu_{i} \mathbf{v}_{j}+\ldots, \frac{d \mathbf{P}}{d t}=\mathbf{0}
$$

and if the center of mass is the origin, the position of the Sun has to be corrected (to indicate the Sun we use the subscript 0):

$$
\mathbf{r}_{0}=\frac{-1}{\mu_{0}\left(1-\eta \Omega_{0}\right)} \sum_{j \neq 0}\left(1-\eta \Omega_{j}\right) \mu_{j} \mathbf{r}_{j}+\cdots
$$

The partial derivative of the acceleration of the body $j$ with respect to $\eta$ is

$\frac{\partial}{\partial \eta} \frac{d^{2} \mathbf{r}_{j}}{d t^{2}}=\Omega_{j}\left[\frac{\mu_{0}}{r_{j 0}^{3}} \mathbf{r}_{j 0}+\sum_{i \neq j, 0} \frac{\mu_{i}}{r_{j i}^{3}} \mathbf{r}_{j i}\right]+\frac{\partial \mathbf{r}_{0}}{\partial \eta} \frac{\partial\left(\mu_{0} / r_{j 0}^{3}\right)}{\partial \mathbf{r}_{0}}$,

where the first term is the direct $\eta$-perturbation, the second the indirect, and

$$
\frac{\partial \mathbf{r}_{0}}{\partial \eta}=\sum_{i \neq 0}\left(\Omega_{j}-\Omega_{0}\right) \frac{\mu_{i}}{\mu_{0}} \mathbf{r}_{i}
$$

By combining together and omitting smaller terms with $\Omega_{i} \mu_{k}$ (with $i, k \neq 0$ ) or $\mathcal{O}\left(\eta^{2}\right)$

$$
\frac{\partial}{\partial \eta} \frac{d^{2} \mathbf{r}_{j}}{d t^{2}}=\Omega_{j} \mu_{0} \frac{\mathbf{r}_{j 0}}{r_{j 0}^{3}}-\Omega_{0} \frac{\partial\left(1 / r_{j 0}^{3}\right)}{\partial \mathbf{r}_{0}} \sum_{i \neq 0} \mu_{i} \mathbf{r}_{i},
$$

with a direct (small parameter $\Omega_{j} \mu_{0}$ ) and an indirect (small parameter $\Omega_{0} \mu_{i}$ ) part.

\section{A. ORBIT14 software structure}

Since the real data from the spacecraft will be available only in 2025, the first program of the software is the simulator, which generates fictitious sets of observables, nongravitational accelerations and initial orbital elements for the probe at the central time of each observed arc. These are obtained by propagating the orbit of the spacecraft starting from some initial conditions taken by the spice kernel generated by the navigation team of the mission.

The core of the orbit determination software is the corrector, the purpose of which is to estimate the parameters we are interested in. This program is the one that will be used to analyze real data. The corrector follows a classical approach (see, for instance, [17]), and its aim is to perform a nonlinear least-squares fit to compute a set of parameters $\mathbf{q}^{*}$ which minimizes the target function

$$
Q(\mathbf{q})=\frac{1}{m} \boldsymbol{\xi}^{T}(\mathbf{q}) \mathbf{W} \boldsymbol{\xi}(\mathbf{q})=\frac{1}{m} \sum_{i=1}^{m} w_{i} \xi_{i}^{2}(\mathbf{q}),
$$

where $m$ is the number of observations and $\boldsymbol{\xi}=\mathcal{O}-\mathcal{C}$ is the vector of residuals, the difference between the observed quantities $\mathcal{O}$ and the predicted ones $\mathcal{C}(\mathbf{q})$, computed using suitable models and assumptions. In our case, the observed quantities are range and range-rate data, while the computed observables are the results of the light-time computation (see [16] for more details) as a function of all the quantities $\mathbf{q}$ we want to estimate ( $w_{i}$ is the weight associated to the $i$-observation).

The procedure to compute $\mathbf{q}^{*}$ is based on a modified Newton's method known in the literature as the "differential corrections method"; see, e.g., [17]. Let us define

$$
\mathbf{B}=\frac{\partial \boldsymbol{\xi}}{\partial \mathbf{q}}(\mathbf{q}), \quad \mathbf{C}=\mathbf{B}^{\mathrm{T}} \mathbf{W B},
$$

which are called the design matrix and the normal matrix, respectively. Then the correction,

$$
\Delta \mathbf{q}=\mathbf{C}^{-1} \mathbf{D} \quad \text { with } \quad \mathbf{D}=-\mathbf{B}^{T} \mathbf{W} \boldsymbol{\xi},
$$

is applied iteratively until either $Q$ does not change meaningfully from one iteration to the other or $\Delta \mathbf{q}$ becomes smaller than a given tolerance.

Concerning the observations, we propose to have two ground stations, one observing in the $K_{a}$-band at the Goldstone Deep Space Communications Complex in California (United States) and the other in $X$-band at the Cebreros station in Spain. With this scenario, the observations are split into arcs, with interruptions of tracking not exceeding $1 \mathrm{~h}$, namely, the "observed arcs," with a duration 
from 14 to $19 \mathrm{~h}$. The arcs are separated by intervals in the dark lasting from 5 to $10 \mathrm{~h}$. We call an "extended arc" an observed arc broadened out from half of the dark period before it to half of the dark period after it.

In order to estimate the parameters we do not use a classical multiarc strategy [18], as described for example in [17], but a constrained multiarc strategy. This method is established on the idea that each observed arc belongs to the same object (the spacecraft) and thus the orbits corresponding to two subsequent extended arcs should coincide at the connection time in the middle of the nonobserved interval (see [19] for more details).

\section{B. Nominal simulation}

The simulation scenario (the same as described in [20]) consists of a 365-arc simulation, which corresponds to about $1 \mathrm{yr}$, starting on March 27, 2025. The main assumptions made are briefly described as follows:

(i) Two ground stations are available for tracking, one at the Goldstone Deep Space Communications Complex (California, USA) for the $K_{a}$-band and the other in Spain, at Cebreros station, for the $X$-band. Range measurements are taken every $120 \mathrm{~s}$ and range rate every $30 \mathrm{~s}$, both with top accuracies.

(ii) We impose the Nordvedt equation [21]; i.e., we assume a metric theory to remove the approximate symmetry between $\beta$ and $J_{2 \odot}$,

$$
\eta=4(\beta-1)-(\gamma-1)-\alpha_{1}-\frac{2}{3} \alpha_{2} .
$$

The MORE relativity experiment consists in solving for the following parameters: the PN and related parameters $\left(\gamma, \beta, \eta, \alpha_{1}, \alpha_{2}, J_{2 \odot}, \mu_{\odot}, \zeta\right)$ plus six initial conditions for the Mercury barycenter $\left\{X_{m}, Y_{m}, Z_{m}, \dot{X}_{m}, \dot{Y}_{m}, \dot{Z}_{m}\right\}$ and six for the EMB $\left\{X_{e}, Y_{e}, Z_{e}, \dot{X}_{e}, \dot{Y}_{e}, \dot{Z}_{e}\right\}$ with respect to the Solar System Barycenter (SSB) in the Ecliptic J2000 reference frame [22]. Due to rank deficiency, among the 12 parameters (positions and velocities), only 8 can be determined simultaneously. They are the position and velocity of Mercury and two components of the velocity of the EMB.

We solve for all the parameters listed above in a global least-squares fit. We performed both an analysis based on formal statistics (standard deviations and correlations) as given from the formal covariance matrix $\boldsymbol{\Gamma}=\mathbf{C}^{-1}$ and an analysis based on "true" errors, defined as the difference between the values of the parameters at convergence and the simulated values. We perform a statistical analysis over ten runs, each time varying the random generator of Gaussian distribution, and we consider as the true error the distribution mean value. Concerning the convergence requirements, we imposed a tolerance threshold in target function variation between two subsequent iterations of $10^{-4}$, and we verified that the differential correction process always reached this condition in six iterations.
TABLE I. Results for RMS of PN and related parameters obtained with ORBIT14 numerical simulations in the "ideal" case (planetary masses have no errors).

\begin{tabular}{lll}
\hline \hline Parameter & Units & Formal sigma \\
\hline$X_{m}$ & $\mathrm{~cm}$ & 0.49 \\
$Y_{m}$ & $\mathrm{~cm}$ & 1.12 \\
$Z_{m}$ & $\mathrm{~cm}$ & 2.93 \\
$\dot{X}_{m}$ & $\mathrm{~cm} \mathrm{~s}^{-1}$ & $3.54 \times 10^{-7}$ \\
$\dot{Y}_{m}$ & $\mathrm{~cm} \mathrm{~s}^{-1}$ & $2.81 \times 10^{-7}$ \\
$\dot{Z}_{m}$ & $\mathrm{~cm} \mathrm{~s}^{-1}$ & $1.43 \times 10^{-6}$ \\
$\dot{X}_{e}$ & $\mathrm{~cm} \mathrm{~s}^{-1}$ & $1.43 \times 10^{-7}$ \\
$\dot{Y}_{e}$ & $\mathrm{~cm} \mathrm{~s}^{-1}$ & $2.76 \times 10^{-7}$ \\
$\beta$ & & $6.21 \times 10^{-7}$ \\
$\gamma$ & & $7.65 \times 10^{-7}$ \\
$\eta$ & & $\mathbf{1 . 9 3} \times \mathbf{1 0}^{-\mathbf{6}}$ \\
$\alpha_{1}$ & & $4.5 \times 10^{-7}$ \\
$\alpha_{2}$ & & $7.6 \times 10^{-8}$ \\
$\mu_{0}$ & & $3.9 \times 10^{13}$ \\
$J_{2 \odot}$ & $\mathrm{cm}^{3} / \mathrm{s}^{2}$ & $3.8 \times 10^{-10}$ \\
$\zeta=\dot{\mu}_{0} / \mu_{0}$ & & $2.0 \times 10^{-14}$ \\
\hline \hline
\end{tabular}

The results for the PN and related parameters in terms of formal uncertainty are shown in Table I.

The parameters $\eta$ and $\zeta$ present true errors always higher than formal ones because they are very sensitive to the effect of systematic errors in range. Nevertheless, the expected results of MORE could hence improve the actual knowledge, even if there are some intrinsic problems due to the uncertainties in the masses and ephemerides of Solar System bodies (see the next section and [23]).

\section{ANALYTICAL MODEL AND SOURCES OF UNCERTAINTIES}

ORBIT14 integrates the orbits of EMB and Mercury, while the trajectories of planets and asteroids are taken from JPL ephemerides. The position of the Sun is obtained from Eq. (5) as a function of positions and (relativistic) masses of the other bodies.

We want to test how the spurious signal due to a wrong value of the mass of a planet affects the estimation of $\eta$. This kind of test cannot be performed by ORBIT14. In fact, we could try to fit the simulated data by using a model with a slightly different value of the mass. At the first iteration, the modeled barycentric orbits of the planets are the same as those of the data, since they come from ephemerides, but the position of the Sun is slightly different due to the different mass of the planet. Hence, all mutual distances among Solar System bodies are altered and the modeled MPO-Earth range turns out to be very different from the simulated data. This implies an unphysical systematic effect on the parameters' estimation. The parameter $\eta$ heavily feels this effect because it enters into the relativistic equation of the center of mass Eq. (5). 
To perform this test, we develop a heliocentric analytical model and we include in our calculus the parameters whose signals are expected to be correlated with the SEP violation signature. In order to avoid systematic effects, GPs must be added to the set of parameters to be fitted and their errors must be taken into account in terms of constraints (hereafter a priori) to be included to the global covariance analysis. All signals involved in the relativity experiment have frequencies of the same order of planetary mean motions. For this reason, we can neglect the motion of MPO around Mercury (the orbital period is approximately $2 \mathrm{~h}$ ) and we will consider the Mercury-Earth range. Current uncertainties of planetary GPs go from $2.8 \times 10^{-4}$ (Mars) to $\approx 10.5 \mathrm{~km}^{3} / \mathrm{s}^{2}$ (Neptune) [24]. Regarding asteroids, relative errors can be very large (50\% or more).

To summarize, we will calculate the signatures on the Earth-Mercury range due to

(1) initial conditions of Earth and Mercury,

(2) SEP violation (parameter $\eta$ ),

(3) planets/dwarf planets/asteroids (GPs to be fitted),

(4) secular variation of Sun's GP $\mu_{0}$ [parameters to be fitted are $\delta_{\mu_{0}}$ (displacement from the nominal GP of the Sun at the starting epoch) and its rate of change in time $\left.\zeta=\dot{\mu}_{0} / \mu_{0}\right]$,

(5) $\mathrm{PN}$ parameter $\bar{\beta}=\beta-1$,

(6) Sun's quadrupole coefficient $J_{2 \odot}$.

The parameter $\gamma$, which is related to the curvature produced by unit rest mass, has not been considered for simplicity. However, this is not reductive since the best estimation of $\gamma$ is expected to be given after the dedicated superior conjunction experiment (SCE) and its RMS $\sigma[\gamma]=2.0 \times$ $10^{-6}$ will be inserted as an a priori into the Nordtvedt equation.

\section{A. Analytical model}

We adopt the notation of [25]: we define $\mathbf{r}_{i j}=\mathbf{r}_{j}-\mathbf{r}_{i}$ and $r_{i j}=\left\|\mathbf{r}_{i j}\right\|$, where $\mathbf{r}_{i}$ is the coordinate of planet $i$ in an inertial reference frame. Planets are numbered from 1 (Mercury) to 8 (Neptune), while 0 is referred to the Sun. We will use subscripts/superscripts $i$ and $k$ to indicate Mercury and Earth, respectively, while $j$ will be used for an arbitrary perturber body (planet or asteroid). We call $\mathbf{q}=\left\{q_{1}, \ldots, q_{N}\right\}$ the set of parameters to be fitted and $\rho_{13}(t, \mathbf{q})$ the analytical model of the Mercury-Earth range to be calculated as a function of $\mathbf{q}$. We will describe the motion of the planet $i$ as a small perturbation from a heliocentric circular orbit with radius $R_{0 i}$ equal to the semimajor axis and mean motion $n_{i}=\left[\left(\mu_{0}+\mu_{i}\right) / R_{0 i}^{3}\right]^{1 / 2}$. For all parameters except $J_{2 \odot}$ (see below) inclinations will be neglected, while eccentricities are assumed to be zero in all cases. The displacement from the reference orbit is $\delta \mathbf{r}_{i}=x_{i} \mathbf{u}_{r}^{i}+y_{i} \mathbf{u}_{t}^{i}+z_{i} \mathbf{u}_{z}^{i}$, where $\mathbf{u}_{r}^{i}, \mathbf{u}_{t}^{i}, \mathbf{u}_{z}^{i}$ are radial, along-track, and out-of-plane unit vectors, respectively. We will express the position of $k$ relative to $i$ as

$$
\mathbf{r}_{i k}=\mathbf{R}_{i k}+\sum_{m=1}^{N} q_{m} \delta \mathbf{r}_{i k, m}
$$

where $\mathbf{R}_{i k}=R_{0 k} \mathbf{u}_{r}^{k}-R_{0 i} \mathbf{u}_{r}^{i}$.

Since terms in the summation are small, at the first order the range is

$$
\rho_{i k}=\left\|\mathbf{r}_{i k}\right\| \approx R_{i k}+\sum_{n} q_{n} \frac{\delta \mathbf{r}_{i k, n} \cdot \mathbf{R}_{i k}}{R_{i k}} .
$$

The factor $1 / R_{i k}$ can be expressed by the Legendre polynomials $P_{n}$ (for $R_{0 i}<R_{0 k}$ ) [26],

$$
\frac{1}{R_{i k}}=\frac{1}{R_{0 k}} \sum_{n=0}^{\infty}\left(\frac{R_{0 i}}{R_{0 k}}\right)^{n} P_{n}\left(\cos \Phi_{i k}\right),
$$

where we defined $\Phi_{i}=n_{i} t+\varphi_{i}$ and $\Phi_{i k}=\left(n_{k}-n_{i}\right) t+$ $\varphi_{k}-\varphi_{i}$. Afterwards, for each $q_{n}$ we will calculate the corresponding $\delta \mathbf{r}_{i k, m}$.

We decompose the perturbation on $i$ (or $k$ ) as a sum of radial and along-track forces $\sum_{n} q_{n}{ }^{\prime}\left(R_{n}^{i} \mathbf{u}_{r}^{i}+T_{n}^{i} \mathbf{u}_{t}^{i}\right)$, where $\mathbf{q}^{\prime}$ represents the subset of $N-12$ dynamical parameters to be estimated (all but initial conditions of Earth and Mercury). For simplicity we assume that perturbations are on the ecliptic plane.

Since we are assuming that $\left\|\delta \mathbf{r}_{i}\right\| \ll R_{0 i}$, we can use the first order Hill's equations [27]. They are

$$
\begin{aligned}
\ddot{x}_{i}-2 n_{i} \dot{y}_{i}-3 n_{i}^{2} x_{i} & =\sum_{n} q_{n}^{\prime} R_{n}^{i} \\
\ddot{y}_{i}+2 n_{i} \dot{x}_{i} & =\sum_{n} q_{n}^{\prime} T_{n}^{i} \\
\ddot{z}_{i}+n_{i}^{2} \dot{z}_{i} & =0 .
\end{aligned}
$$

Solutions are the sum of the homogeneous part $\left\{\hat{x}_{i}, \hat{y}_{i}, \hat{z}_{i}\right\}$ plus the contributions $\left\{x_{i}^{\prime}, y_{i}^{\prime}, z_{i}^{\prime}\right\}$ due to the perturbing forces. Since Eqs. (16) are linear, the inhomogeneous terms can be calculated one at a time and finally summed together.

\section{Initial conditions}

Here we calculate the signature on range due to initial conditions of Earth and Mercury. We consider two cases: initial conditions expressed in heliocentric and in barycentric (SSB) reference frame. In this latter case an additional signal which depends on $\eta$ must be included.

Referring to Eq. (16), we express the state vector $\mathbf{v}=\left\{x_{i}, y_{i}, z_{i}, \dot{x}_{i}, \dot{y}_{i}, \dot{z}_{i}\right\}$ of body $i$ as

$$
\mathbf{v}=\hat{\mathbf{v}} \mathbf{A}+\mathbf{v}^{\prime} \mathbf{q}^{\prime}
$$

where the first term on the right side represents the homogeneous solution of Eq. (16). It is the product of a $6 \times 6$ matrix $\hat{\mathbf{v}}$ [see Eq. (A1) in the Appendix] and the 
vector $\mathbf{A}$ of six coefficients to be fixed by the initial conditions. The second term, the inhomogeneous solution, is the product of the $6 \times(N-12)$ matrix $\mathbf{v}^{\prime}$ of the particular solutions and the column vector $\mathbf{q}^{\prime}$ of the parameters $q_{n}^{\prime}$.

We pass to the coordinate system with fixed axes and we express the state vector as $\mathbf{v}=\mathcal{R} \delta \mathbf{x}$, where $\mathcal{R}$ is the corresponding rotation matrix [see Eq. (A3)]. We rewrite $\mathbf{A}$ in terms of $\delta \mathbf{x}$ and $\mathcal{R}$ at $t=0$ (say $\delta \mathbf{x}_{0}, \mathcal{R}_{0}$ ) and, defining $\mathcal{V}=\hat{\mathbf{v}} \hat{\mathbf{v}}_{0}^{-1}$ [see Eq. (A2)], we get

$$
\mathbf{v}=\mathcal{V} \mathcal{R}_{0} \delta \mathbf{x}_{0}+\left(\mathbf{v}^{\prime}-\mathcal{V} \mathbf{v}_{0}^{\prime}\right) \mathbf{q}^{\prime}
$$

The first term represents the "signal" due to initial conditions $\delta \mathbf{x}_{0}$, while the signal due to parameter $q_{n}^{\prime}$ (the term in parentheses) is the sum of the particular solution $\mathbf{v}^{\prime}$ plus the homogeneous solution corresponding to $\delta \mathbf{x}_{0}=0$. The complete set of parameters to be determined by the ranging between $i$ and $k$ is

$$
\mathbf{q}=\left\{\delta \mathbf{x}_{0}^{i}, \delta \mathbf{x}_{0}^{k}, \mathbf{q}^{\prime}\right\}
$$

Defining the $6 \times 6$ matrix $f_{\alpha \mu}^{i}$ and $6 \times(N-12)$ matrix $g_{\alpha \mu}^{i}$ as

$$
f_{\alpha \mu}^{i}=\mathcal{R}^{-1} \mathcal{V} \mathcal{R}_{0} ; \quad g_{\alpha \mu}^{i}=\mathcal{R}^{-1}\left(\mathbf{v}^{\prime}-\mathcal{V} \mathbf{v}_{0}^{\prime}\right)
$$

$(\mathcal{R}, \mathcal{V}$, and $\mathbf{v}$ are referred to body $i$ ) and using $\alpha=1,2,3$ to indicate the spatial components in the fixed-axes coordinate system of $\delta \mathbf{r}_{i k, m}$, we get

$\delta \mathbf{r}_{i k, m}= \begin{cases}-f_{\alpha \lambda}^{i} & \lambda=m \quad \text { and } \quad m \leq 6 ; \\ f_{\alpha \lambda}^{k} & \lambda=m-6 \text { and } 7 \leq m \leq 12 ; \\ g_{\alpha \lambda}^{k}-g_{\alpha \lambda}^{i} & \lambda=m-12 \text { and } \quad m>12 .\end{cases}$

Finally, by Eqs. (14), (21) we obtain the perturbation on range due to each element of $\mathbf{q}$.

The barycentric initial state vector $\mathbf{X}_{0}^{i}$ is related to the heliocentric one by

$$
\mathbf{X}_{0}^{i}=\left(1+\eta \Omega_{0}\right) \mathbf{R}_{0}+R_{0 i} \mathbf{s}_{i}+\delta \mathbf{x}_{0}^{i},
$$

where

$$
\mathbf{s}_{j}=\left\{\cos \varphi_{j}, \sin \varphi_{j}, 0,-n_{j} \sin \varphi_{j}, n_{j} \cos \varphi_{j}, 0\right\}
$$

and

$$
\mathbf{R}_{0}=-\frac{\sum_{j \neq 0} \mu_{j} R_{0 j} \mathbf{s}_{j}}{\sum_{j} \mu_{j}}
$$

is the position of the Sun with respect to the SSB in the case $\eta=0$. Therefore, if we pass to barycentric initial conditions we must take into account additional signals due to $\mu_{j}$ 's and $\eta$.
By Eq. (22) we can express Eq. (18) as a function of $\mathbf{X}_{0}^{i}$. Adopting now $\mathbf{q}=\left\{\mathbf{X}_{0}^{i}, \mathbf{X}_{0}^{k}, \mathbf{q}^{\prime}\right\}$, we calculate the extra signals due to $\eta$ and $\mu_{l}$ to be added to $\delta \mathbf{r}_{i k, m}$ in Eq. (21). They are reported in Eq. (B1).

\section{SEP violation and contributions of planets/asteroids}

In the heliocentric reference frame, the equations of motion of a planet $i$, in the case $\eta \neq 0$, are $[2,4,11,26,28]$

$\ddot{\mathbf{r}}_{0 i}=-\frac{\mu^{\star}}{r_{0 i}^{3}} \mathbf{r}_{0 i}+\sum_{j \neq i \neq 0} \mu_{j}\left[\left(1+\eta \Omega_{i}\right) \frac{\mathbf{r}_{i j}}{r_{i j}^{3}}-\left(1+\eta \Omega_{0}\right) \frac{\mathbf{r}_{0 j}}{r_{0 j}^{3}}\right]$,

where the summation is extended to all Solar System bodies and

$$
\mu^{\star}=\mu_{0}+\mu_{i}+\eta\left(\mu_{i} \Omega_{0}+\mu_{0} \Omega_{i}\right) .
$$

From Eq. (25) a high correlation among planetary perturbations (depending on $\mu_{j}$ ) and SEP violation is evident.

We separate the contributions of parameters $\eta$ and $\mu_{j}$ and we project them on radial and along-track directions. Since $\Omega_{i} \ll \Omega_{0}$, the SEP violation contribution can be simplified $[4,23,26]$ :

$$
\begin{aligned}
& R_{\eta}^{i} \approx-\Omega_{i} n_{i}^{2} R_{0 i}-\Omega_{0} \sum_{j \neq i \neq 0} \frac{\cos \Phi_{i j}}{R_{0 j}^{2}} ; \\
& T_{\eta}^{i} \approx-\Omega_{0} \sum_{j \neq i \neq 0} \frac{\sin \Phi_{i j}}{R_{0 j}^{2}}
\end{aligned}
$$

[see Eq. (C1) for the complete expression].

The signal contains a small permanent radial displacement due to a "direct" term $\propto \Omega_{i}$ and an "indirect" term, which depend on $\Omega_{0}$. These terms have been calculated in the SSB frame in Sec. II [see Eq. (8)].

The particular solution of Eq. (16) relative to parameter $\eta$ can be written as $\left\{x_{i, \eta}^{\prime}, y_{i, \eta}^{\prime}\right\}$, where

$$
\begin{aligned}
& x_{i, \eta}^{\prime}=\Omega_{i} \frac{R_{0 i}}{3}+\Omega_{0} \sum_{j \neq i} \frac{\mu_{j}}{R_{0 j}^{2}} \frac{1+2 n_{i} / n_{j i}}{n_{j i}^{2}-n_{i}^{2}} \cos \Phi_{j i}, \\
& y_{i, \eta}^{\prime}=-\Omega_{0} \sum_{j \neq i} \frac{\mu_{j}}{R_{0 j}^{2}} \frac{1+2 n_{i} / n_{j i}+3 n_{i}^{2} / n_{j i}^{2}}{n_{j i}^{2}-n_{i}^{2}} \sin \Phi_{j i} .
\end{aligned}
$$

Similarly, perturbations on planet $i$ due to body $j$ are (radial and along-track) [26]

$$
\begin{aligned}
R_{\mu_{j}}^{i} & =\sum_{j \neq i \neq 0}\left(\frac{R_{0 j} \cos \Phi_{i j}-R_{0 i}}{R_{i j}^{3}}-\frac{\cos \Phi_{i j}}{R_{0 j}^{2}}\right), \\
T_{\mu_{j}}^{i} & =\sum_{j \neq i \neq 0}\left(\frac{R_{0 j}}{R_{i j}^{3}}-\frac{1}{R_{0 j}^{2}}\right) \sin \Phi_{i j} .
\end{aligned}
$$


The coefficient $1 / R_{i j}^{3}$ can be calculated from Eq. (15) and expressed as a Fourier cosine series with fundamental frequency $\Phi_{i j}$ [see Eq. (D1)]. Therefore, we can write

$$
\begin{aligned}
R_{\mu_{j}}^{i} & =\sum_{l=0}^{\infty} a_{j, l} \cos \left(l \Phi_{i j}\right) ; \\
T_{\mu_{j}}^{i} & =\sum_{l=1}^{\infty} b_{j, l} \sin \left(l \Phi_{i j}\right) ;
\end{aligned}
$$

and coefficients $a_{j, l}$ and $b_{j, l}$ are reported in Eq. (D2).

The radial and along-track components of $\left\{x_{i, \mu_{j}}^{\prime}, y_{i, \mu_{j}}^{\prime}\right\}$ are, respectively,

$$
\begin{aligned}
x_{i, \mu_{j}}^{\prime}= & -\frac{a_{j 0}}{3 n_{i}^{2}}-\sum_{l=1}^{\infty} \frac{a_{j, l}-2 b_{j, l} /\left(\ln _{j i}\right)}{l^{2} n_{j i}^{2}-n_{i}^{2}} \cos \Phi_{j i} ; \\
y_{i, \mu_{j}}^{\prime}= & -\sum_{l=1}^{\infty} \frac{b_{j, l}-2 a_{j, l} n_{i} /\left(\ln _{j i}\right)+3 b_{j, l} n_{i}^{2} /\left(l^{2} n_{j i}^{2}\right)}{l^{2} n_{j i}^{2}-n_{i}^{2}} \\
& \times \sin \Phi_{j i} .
\end{aligned}
$$

Finally, by applying Eq. (14) and Eq. (21), the EarthMercury range perturbations due to parameters $\eta$ can be written as

$$
\begin{aligned}
\delta \rho_{13}^{\eta}= & \sum_{l=1}^{\infty} \mathcal{D}_{l} \cos \left(l \Phi_{13}\right) \\
& +\sum_{\substack{j=\mathrm{pl} \\
\text { tast }}} \sum_{\substack{p, q, \mathrm{q} r \\
\in \mathbb{Z}}} \mathcal{I}_{j}^{p q r} \cos \left(p \Phi_{1}+q \Phi_{3}+r \Phi_{j}\right),
\end{aligned}
$$

where coefficients $\mathcal{D}_{l}$ are due to the "direct effect" and they depend only on $\Omega_{1}$ and $\Omega_{3}$.

For all perturbing bodies considered, we calculated the numerical values of amplitudes $\mathcal{D}$ and $\mathcal{I}$ using the complete expression Eq. (C1). They are reported (for planets from Mars to Neptune) in Table IV.

An analog expression can be written for the perturbation due to body $j$,

$$
\delta \rho_{13}^{\mu_{j}}=\sum_{\substack{p, q, r \\ \in \mathbb{Z}}} \mathcal{J}^{p q r} \cos \left(p \Phi_{1}+q \Phi_{3}+r \Phi_{j}\right)
$$

Numerical coefficients for amplitudes $\mathcal{J}$ are reported in Table V.

\section{Range signature due to a variation of Sun's GP}

It is well known that the GP of the Sun is not constant in time due to the Sun's mass loss and to a possible (but unconfirmed) dependence of $G$ on time. Mathematically this corresponds to

$$
G M_{\odot}(t)=\mu_{0}\left[1+\zeta\left(t-t_{0}\right)\right]+\delta_{\mu_{0}},
$$

where $t_{0}$ is the epoch (hereafter $t_{0}=0$ ) when the GP of the Sun is equal to $\mu_{0}+\delta_{\mu_{0}}$. The small parameter $\delta_{\mu_{0}}$ has been introduced to account for the fact that the true value and nominal value $\mu_{0}$ of the GP of the Sun are not the same. The parameter $\zeta=\dot{\mu}_{0} / \mu_{0}$ is a small (constant) rate of change.

If $\zeta \neq 0$ and/or $\mu_{0}$ is slightly different from the nominal value, a radial perturbation will be present. The Hill's equations are

$$
\begin{aligned}
\ddot{x}_{i}-2 n_{i} \dot{y}_{i}-3 n_{i}^{2} x_{i} & =-\frac{\delta_{\mu_{0}}+\mu_{0} \zeta t}{R_{0 i}^{3}}, \\
\ddot{y}_{i}+2 n_{i} \dot{x}_{i} & =0,
\end{aligned}
$$

and a particular solution is

$$
x_{i}^{\prime}=-\frac{\delta_{\mu_{0}}+\mu_{0} \zeta t}{n_{i}^{2} R_{0 i}^{2}} ; \quad y_{i}^{\prime}=\frac{\mu_{0} \zeta t^{2}+2 \delta_{\mu_{0}} t}{n_{i} R_{0 i}^{2}} .
$$

By Eqs. (14), (21) we obtain the range signature due to $\zeta$ and $\delta_{\mu_{0}}$.

\section{Range signature due to $\beta \neq 1$}

The PN parameter $\beta$ is related to the nonlinearity in the superposition of gravity. In GR it is, by definition, $\beta=1$. Defining the small parameter $\bar{\beta}=\beta-1$, the perturbing force per unit mass on body $i$ in an inertial frame is

$$
\mathbf{a}_{\beta}^{i}=-\frac{2 \bar{\beta}}{c^{2}} \sum_{j \neq i} \frac{\mu_{j} \mathbf{r}_{i j}}{r_{i j}^{3}}\left[\sum_{h \neq i} \frac{\mu_{h}}{r_{i h}}+\sum_{k \neq j} \frac{\mu_{k}}{r_{j k}}\right] .
$$

We calculate it in the heliocentric frame $\mathbf{a}_{\beta}^{i}-\mathbf{a}_{\beta}^{0}$. The biggest term is $2 \bar{\beta} / c^{2} \mu_{0}^{2} / R_{0 i}^{3} \mathbf{u}_{r}^{i}$ and all others are at least 3 orders of magnitude smaller.

The effect due to $\bar{\beta}$ is essentially a radial force, as for $\delta_{\mu_{0}}$ and $\zeta$. A particular solution is

$$
x_{i}=\bar{\beta} \frac{2 \mu_{0}^{2}}{R_{0 i}^{3} n_{i}^{2} c^{2}}, \quad y_{i}=-\bar{\beta} \frac{4 \mu_{0}^{2}}{R_{0 i}^{3} c^{2} n_{i}} t,
$$

and by Eqs. (14), (21) we obtain the range signature for $\bar{\beta}$.

\section{Range signature due to Sun's $\boldsymbol{J}_{2 \odot}$}

So far, inclinations have been neglected: all planets are assumed to orbit on the ecliptical plane, but in this case we will consider the orbital inclinations with respect to the Sun's equatorial plane. This is necessary to avoid a fictitious strong correlation between $J_{2 \odot}$ and $\bar{\beta}, \mu_{0}$ or $\zeta$.

Inclinations of Mercury and Earth orbits with respect to the Sun's equator are $3.380^{\circ}$ and $7.155^{\circ}$ respectively. Unit vectors $\mathbf{u}_{r}, \mathbf{u}_{t}$, and $\mathbf{u}_{z}$ have been rewritten to take into account the orbital elements of the planet.

The perturbation of the Sun's $J_{2 \odot}$ on the trajectory of a planet can be obtained by solving this set of equations [34]: 


$$
\begin{aligned}
\ddot{x}-2(n c) \dot{y}-\left(5 c^{2}-2\right) n^{2} x & =-3 \alpha n^{2}(3+5 s) \cos (2 n c t+\varphi) \\
\ddot{y}+2(n c) \dot{x} & =-2 \alpha n^{2}(3+5 s) \sin (2 n c t+\varphi) \\
\ddot{z}+\left(3 c^{2}-2\right) n^{2} z & =-2 \beta n^{2} s \sqrt{1+3 s} \sin (n c t+\varphi),
\end{aligned}
$$

where

$c=\sqrt{1+s} ; \quad s=\frac{3 J_{2 \odot} R_{\odot}^{2}}{8 r^{2}}[1+3 \cos (2 I)] ;$

$\alpha=\frac{3 J_{2 \odot} R_{\odot}^{2}}{8 r(3+5 s)}[1-\cos (2 I)] ; \quad \beta=\frac{3 J_{2 \odot} R_{\odot}^{2}}{4 r s \sqrt{1+3 s}} \sin (2 I) ;$

and $I$ is the inclination, $R_{\odot}$ is the radius of the Sun, $n$ is the mean motion, $r$ is the Sun-planet distance, and $\varphi$ is the initial phase.

Hill's equations have been modified by increasing the angular velocity of the reference frame from $n$ up to $n c$ in order to avoid drifts into the inhomogeneous solutions (see [34] for details). The particular solution is

$$
\begin{aligned}
& x^{\prime}=\alpha \cos (2 n c t+\varphi), \\
& y^{\prime}=\alpha \frac{1+3 s}{2(1+s)} \sin (2 n c t+\varphi), \\
& z^{\prime}=-\beta \sqrt{1+3 s} \sin (n c t+\varphi) .
\end{aligned}
$$

Since Eqs. (41) are not linear functions of $J_{2 \odot}$, we expand them as MacLaurin series of $J_{2 \odot}$ up to the first order (for Mercury and Earth).

By Eqs. (14) and (21) we estimate the perturbation on the range due to $J_{2 \odot}$.

\section{Time sampling}

Due to the visibility windows, as described in Sec. II A, range and range-rate data contain several gaps. A gap occurs approximately at each arc and lasts about $9.3 \mathrm{~h}$.

To perform a realistic calculus, we evaluate the perturbations at the set of epochs $t_{i}$ (spanning an interval of 373 d) generated by ORBIT14. A low-frequency sampling $\left(f_{s}=10^{-4} \mathrm{~Hz}\right)$ is sufficient for our purposes since the signals involved have frequencies of the same order of planetary mean motions. For the RMS $\hat{\sigma}_{i}$ relative to the $i$ th range data, we adopt [17]

$$
\hat{\sigma}_{i}=15 \mathrm{~cm} \sqrt{300 f_{s}} \approx 2.6 \mathrm{~cm} .
$$

\section{Constraints and covariance matrix calculus}

As explained in Sec. II B, there is a subset of $M$ parameters $\mathbf{x}=\left\{x_{1}, \ldots, x_{M}\right\} \in \mathbf{q}$ for which information is available from other experiments. In our case they are the GPs and $\gamma$. The information about $\gamma$ from SCE affects $\beta$ and $\eta$ thanks to the Nordtvedt equation.
We define the a priori observations $\mathbf{x}^{P}$ and $\mathbf{C}^{P}$ as the nondiagonal a priori normal matrix used to represent the information available about parameters $\mathbf{x}$. Errors relative to the a priori constraints are $\sigma_{i}$, with $i=1 \ldots M$. The constraint involving the subset $\mathbf{x}$ can be written as $\mathbf{C}^{P} \mathbf{x}=\mathbf{C}^{P} \mathbf{x}^{P}$.

Following [17], we modify Eq. (9) by including the constraints

$$
Q(\mathbf{q})=\frac{1}{m+M}\left[\boldsymbol{\xi}^{T}(\mathbf{q}) \mathbf{W} \boldsymbol{\xi}(\mathbf{q})+\left(\mathbf{x}-\mathbf{x}^{P}\right)^{T} \mathbf{C}^{P}\left(\mathbf{x}-\mathbf{x}^{P}\right)\right] .
$$

In our case we have

$$
\left(\mathbf{x}-\mathbf{x}^{P}\right)^{T} \mathbf{C}^{P}\left(\mathbf{x}-\mathbf{x}^{P}\right)=\frac{(\eta-4 \bar{\beta})^{2}}{\sigma_{N}^{2}}+\sum_{i} \frac{\left(\mu_{i}-\mu_{i}^{P}\right)^{2}}{\sigma_{i}^{2}},
$$

where $\sigma_{N}=2.0 \times 10^{-6}$ is the expected RMS of $\gamma$ after a SCE, while the summation is extended to all GPs and $\sigma_{i}$ are the corresponding errors.

By means of well-known formulas, we obtain the normal matrix $\mathbf{C}$ and its inverse, the covariance matrix $\Gamma$. Finally, the diagonal elements of $\Gamma$ give us the expected RMSs of the parameters.

For a large fraction of asteroids, GPs are estimated by ground-based measurements of diameters and supposed density values; therefore uncertainties are large. However, at the epoch of the BC mission, a certain number of GPs will become more precise thanks to the GAIA mission by precise measurements of the perturbations on Mars's orbit and asteroid-asteroid close approaches.

By DAWN measurements, the error of 4 Vesta's GP is now $1.2 \times 10^{-5} \mathrm{~km}^{3} / \mathrm{s}^{2}$ [33], while the GP of 1 Ceres has been recently estimated with an error of $8.0 \times 10^{-4} \mathrm{~km}^{3} / \mathrm{s}^{2}$ [31]. The current error $\sigma\left[\mu_{5}\right]=2.7 \mathrm{~km}^{3} / \mathrm{s}^{2}$ of the GP of the Jupiter system could be improved by JUNO mission measurements up to $\sigma\left[\mu_{5}\right]=0.53\left(X\right.$-band) or $0.20 \mathrm{~km}^{3} / \mathrm{s}^{2}$ ( $K_{a}$-band) [29]. In [32] a list of asteroids with their expected relative errors on GPs after the GAIA mission was reported; 62 of them belong to the sample of 343 asteroids we considered. We perform tests with both the "current" and the "expected" values (reported in Table II).

\section{Results}

We made a preliminary test to check the results of the analytical model with those of the nominal experiment described in Sec. II B. Therefore, we perform the covariance analysis for the following set of parameters only: barycentric initial conditions of Mercury and Earth plus $\bar{\beta}$, $\eta, \delta_{\mu_{0}}, J_{2 \odot}$ and $\zeta$. Results are reported in column 1 of Table III; by comparing with the RMSs of Table I we find a very good agreement. In particular, the outcome for $\eta$ from 
TABLE II. Current and expected uncertainties for GPs of planets/dwarf planets/asteroids. For Jupiter, the improvement could be reached by JUNO mission data, and for the others in the list, by GAIA.

\begin{tabular}{lccc}
\hline \hline Body & $\begin{array}{c}\text { Current GP } \\
\text { errors }\left(\mathrm{km}^{3} / \mathrm{s}^{2}\right)\end{array}$ & $\begin{array}{c}\text { Expected GP } \\
\text { errors }\left(\mathrm{km}^{3} / \mathrm{s}^{2}\right)\end{array}$ & Refs. \\
\hline Venus & 0.0063 & 0.0063 & {$[24]$} \\
Mars & 0.00028 & 0.00028 & {$[24]$} \\
Jupiter & 2.7 & 0.5 & {$[24,29]$} \\
Saturn & 1.1 & 1.1 & {$[24]$} \\
Uranus & 5.0 & 5.0 & {$[24]$} \\
Neptune & 10.5 & 10.5 & {$[24]$} \\
Pluto & 2.1 & 2.1 & {$[30]$} \\
Eris & 13.1 & 13.1 & {$[24]$} \\
1 Ceres & 0.0008 & 0.0008 & {$[31]$} \\
2 Pallas & 0.28 & 0.17 & {$[24,32]$} \\
3 Juno & 0.11 & 0.037 & {$[24,32]$} \\
4 Vesta & 0.000012 & 0.000012 & {$[33]$} \\
10 Hygiea & 0.48 & 0.043 & {$[32]$} \\
704 Interamnia & 0.47 & 0.11 & {$[32]$} \\
$\ldots$ & $\ldots$ & $\cdots$ & $\cdots$ \\
\hline \hline
\end{tabular}

the analytical model is $\sigma[\eta]=1.58 \times 10^{-6}$, to be compared with $1.93 \times 10^{-6}$ of ORBIT14.

After the validation of the analytical model, we did two experiments with an extended set of parameters. In both cases we added to the list the GPs of planets from Jupiter to Neptune plus Pluto, Eris, and the whole sample of asteroids involved in the dynamics of ORBIT14. The total number of parameters was 362 . The Nordtvedt a priori has been included in both experiments.

TABLE III. RMSs of the parameters obtained by the analytical global covariance analysis. (1) Metric + SCE experiment assuming no errors on GPs (to be compared with numerical results listed in Table I). (2) Metric + SCE experiment assuming current errors on GPs. (3) is as (2) but with expected errors on GPs.

\begin{tabular}{|c|c|c|c|c|}
\hline \multicolumn{5}{|c|}{ Relativity experiment (integration time: 373 d) } \\
\hline Parameter & Units & (1) & (2) & (3) \\
\hline$\overline{X_{m}}$ & $\mathrm{~cm}$ & 0.29 & $2.51 \times 10^{3}$ & $2.49 \times 10^{3}$ \\
\hline$Y_{m}$ & $\mathrm{~cm}$ & 0.88 & $1.19 \times 10^{4}$ & $1.18 \times 10^{4}$ \\
\hline$Z_{m}$ & $\mathrm{~cm}$ & 4.62 & 5.38 & 5.15 \\
\hline$\dot{X}_{m}$ & $\mathrm{~cm} \mathrm{~s}^{-1}$ & $3.91 \times 10^{-7}$ & $2.38 \times 10^{-3}$ & $2.36 \times 10^{-3}$ \\
\hline$\dot{Y}_{m}^{m}$ & $\mathrm{~cm} \mathrm{~s}^{-1}$ & $2.93 \times 10^{-7}$ & $1.70 \times 10^{-3}$ & $1.68 \times 10^{-3}$ \\
\hline$\dot{Z}_{m}^{m}$ & $\mathrm{~cm} \mathrm{~s}^{-1}$ & $3.90 \times 10^{-6}$ & $4.76 \times 10^{-6}$ & $4.72 \times 10^{-6}$ \\
\hline$\dot{X}_{e}$ & $\mathrm{~cm} \mathrm{~s}^{-1}$ & $1.04 \times 10^{-7}$ & $1.79 \times 10^{-3}$ & $1.77 \times 10^{-3}$ \\
\hline$\dot{Y}_{e}$ & $\mathrm{~cm} \mathrm{~s}^{-1}$ & $1.18 \times 10^{-7}$ & $9.47 \times 10^{-5}$ & $9.41 \times 10^{-5}$ \\
\hline$\beta$ & & $6.38 \times 10^{-7}$ & $1.09 \times 10^{-5}$ & $7.81 \times 10^{-6}$ \\
\hline$\eta$ & & $1.58 \times 10^{-6}$ & $4.37 \times 10^{-5}$ & $3.13 \times 10^{-5}$ \\
\hline$\delta_{\mu_{0}}$ & $\mathrm{~cm}^{3} \mathrm{~s}^{-2}$ & $9.19 \times 10^{12}$ & $7.69 \times 10^{13}$ & $5.50 \times 10^{13}$ \\
\hline$J_{2 \odot}$ & & $3.80 \times 10^{-10}$ & $8.49 \times 10^{-10}$ & $8.03 \times 10^{-10}$ \\
\hline$\zeta$ & $\mathrm{yr}^{-1}$ & $1.22 \times 10^{-14}$ & $1.93 \times 10^{-14}$ & $1.78 \times 10^{-14}$ \\
\hline$\cdots$ & & & $\cdots$ & $\cdots$ \\
\hline
\end{tabular}

In the first experiment, we adopt for the a priori's the current errors of GPs, while in the other we use the expected ones, according to the values reported in Table II. The results are reported in columns 2 and 3 of Table III. The resulting RMS of the parameter $\eta$ is about 1 order of magnitude with respect to the ideal case. The difference between the two experiments (current and expected) is small: the a priori's with the expected values improve the RMS of $\eta$ by only a factor of 1.4 , leading to $\sigma[\eta]=3.1 \times 10^{-5}$.

\section{CONCLUSIONS}

In this work we described in detail a semianalytical model for the Earth-Mercury ranging for the BepiColombo mission. Our purpose was the estimation of the RMS of the Nordtvedt parameter $\eta$ by a global covariance analysis. The frequency of signal due to $\eta$ in the Earth-Mercury range is of the same order of planetary mean motions and the parameters that could be in principle correlated with $\eta$ are the initial conditions of Earth and Mercury, the other PN parameters, and the masses of planets and asteroids. We included them in the list of parameters by calculating their signals on the range.

In order to check the analytical model we performed a preliminary covariance analysis, involving 13 parameters, to be compared with the numerical global fit obtained by ORBIT14 in the ideal case of exact knowledge of the masses of planets and asteroids. We found that the RMSs given by our model were in good agreement with those estimated by ORBIT14.

Afterwards, we included in the parameters list the masses of planets and the 343 more massive asteroids (the total number of parameters was 362). The RMSs of the masses have been constrained to their current (or expected at the epoch of the mission) values and we found $\sigma[\eta]=4.37 \times$ $10^{-5}$ and $\sigma[\eta]=3.13 \times 10^{-5}$, respectively. Therefore, the uncertainties of the masses of Solar System bodies degrade the precision of the estimation of $\eta$ by about 1 order of magnitude. However, since the current RMS of $\eta$, from LLR measurements, is $\sigma[\eta]=4.4 \times 10^{-4}$, we conclude that the BepiColombo relativity experiment can improve the current precision on $\eta$ by a factor of 10 .

Finally, the precision of the initial conditions turns out to be very low, but we verified that the uncertainties mostly concern the Sun-SSB position, while the RMS of the heliocentric coordinates remains almost unchanged.

\section{ACKNOWLEDGMENTS}

F. D. M. acknowledges the advice and support of N. Ashby and P. Bender (University of Colorado, Boulder) for fruitful interaction on the development of analytical models. The results of the research presented in this work have been performed within the scope of Contract No. ASI/ 2007/I/082/06/0 with the Italian Space Agency. The authors thank the anonymous referee for his valuable review. 


\section{APPENDIX A: MATRICES}

$$
\begin{gathered}
\hat{\mathbf{v}}=\left(\begin{array}{cccccc}
\cos (n t) & \sin (n t) & 1 & 0 & 0 & 0 \\
-2 \sin (n t) & 2 \cos (n t) & -3 n t / 2 & 0 & 0 & 0 \\
0 & 0 & 0 & 0 & \cos (n t) & \sin (n t) \\
-n \sin (n t) & n \cos (n t) & 0 & 0 & 0 & 0 \\
-2 n \cos (n t) & -2 n \sin (n t) & -3 n / 2 & 0 & 0 & 0 \\
0 & 0 & 0 & 0 & -n \sin (n t) & n \cos (n t)
\end{array}\right) \\
\mathcal{V} \hat{\mathbf{v}}_{0}^{-1}=\left(\begin{array}{ccccccc}
4-3 \cos (n t) & 0 & 0 & \sin (n t) / n & 2[1-\cos (n t)] / n & 0 \\
6[\sin (n t)-n t] & 1 & 0 & 2[\cos (n t)-1] / n & -3 t+4 \sin (n t) / n & 0 \\
0 & 0 & \cos (n t) & 0 & 0 & \sin (n t) / n \\
3 n \sin (n t) & 0 & 0 & \cos (n t) & 2 \sin (n t) & 0 \\
6 n[\cos (n t)-1] & 0 & 0 & -2 \sin (n t) & -3+4 \cos (n t) & 0 \\
0 & 0 & -n \sin (n t) & 0 & 0 & 0 \\
\mathcal{R}= & \sin (n t) & 0 & 0 & 0 & 0 \\
-\cos (n t) & \sin (n t) & \cos (n t) & 0 & 0 & 0 & 0 \\
0 & 0 & 1 & 0 & 0 & 0 \\
-n \sin (n t) & n \cos (n t) & 0 & \cos (n t) & \sin (n t) & 0 \\
-n \cos (n t) & -n \sin (n t) & 0 & -\sin (n t) & \cos (n t) & 0 \\
0 & 0 & 0 & 0 & 0 & 1
\end{array}\right)
\end{gathered}
$$

\section{APPENDIX B: ADDITIONAL SIGNALS DUE TO BARYCENTRIC INITIAL CONDITIONS}

The extra terms to be added to Eq. (21), if initial conditions are barycentric, are $g^{k}-g^{i}$, where

$$
g^{i}=-\mathcal{R}^{-1} \mathcal{V} \mathcal{R}_{0} \begin{cases}\Omega_{0} \mathbf{R}_{0} & q_{m}=\eta ; \\ \left(R_{0 l} \mathbf{s}_{l}+\mathbf{R}_{0}\right) / \mu_{T} & q_{m}=\mu_{l} \neq \mu_{0} ; \\ \mathbf{R}_{0} / \mu_{T} & q_{m}=\mu_{l}=\mu_{0} ;\end{cases}
$$

where $\mu_{T}$ is the total mass (Sun, planets, and asteroids) and $\mathcal{R}, \mathcal{V}, \mathbf{s}_{l}$ are calculated for body $i$.

\section{APPENDIX C: COMPLETE SEP VIOLATION PERTURBING TERM}

Complete radial and along-track perturbations on planet $i$ due to SEP violation:

$$
\begin{aligned}
R_{\eta}^{i} & =-\frac{\mu_{i} \Omega_{0}+\mu_{0} \Omega_{i}}{R_{0 i}^{2}}+\sum_{j \neq i \neq 0} \mu_{j}\left(\Omega_{i} \frac{R_{0 j} \cos \Phi_{i j}-R_{0 i}}{R_{i j}^{3}}-\Omega_{0} \frac{\cos \Phi_{i j}}{R_{0 j}^{2}}\right) \\
T_{\eta}^{i} & =\sum_{j \neq i \neq 0} \mu_{j} \sin \Phi_{i j}\left(\Omega_{i} \frac{R_{0 j}}{R_{i j}^{3}}-\Omega_{0} \frac{1}{R_{0 j}^{2}}\right) .
\end{aligned}
$$




\section{APPENDIX D: COEFFICIENTS FOR PLANETARY PERTURBATIONS}

Series expansion for $1 / R_{i j}^{3}$, where $R_{0 i}<R_{0 j}$ :

$$
\frac{1}{R_{i j}^{3}}=\left(\frac{1}{R_{0 j}^{3}}+\frac{9}{4} \frac{R_{0 i}^{2}}{R_{0 j}^{5}}+\cdots\right)+\left(3 \frac{R_{0 i}}{R_{0 j}^{4}}+\frac{45}{8} \frac{R_{0 i}^{3}}{R_{0 j}^{6}}+\cdots\right) \cos \Phi_{i j}+\left(\frac{15}{4} \frac{R_{0 i}^{2}}{R_{0 j}^{5}}+\frac{105}{16} \frac{R_{0 i}^{4}}{R_{0 j}^{7}}+\cdots\right) \cos 2 \Phi_{i j}+\cdots
$$

Radial $\left(a_{j, l}\right)$ and along-track $\left(b_{j, l}\right)$ coefficients of the perturbation on body $i$ due to body $j$ [see Eq. (30)]:

$$
\begin{aligned}
& a_{j, 0}=\frac{1}{2} \frac{R_{0 i}}{R_{0 j}^{3}}+\frac{9}{16} \frac{R_{0 i}^{3}}{R_{0 j}^{5}}+\frac{75}{128} \frac{R_{0 i}^{5}}{R_{0 j}^{7}} \ldots ; \quad a_{j, 1}=\frac{9}{8} \frac{R_{0 i}^{2}}{R_{0 j}^{4}}+\frac{75}{64} \frac{R_{0 i}^{4}}{R_{0 j}^{6}}+\frac{1225}{1024} \frac{R_{0 i}^{6}}{R_{0 j}^{8}}+\ldots ; \\
& a_{j, 2}=\frac{3}{2} \frac{R_{0 i}}{R_{0 j}^{3}}+\frac{5}{4} \frac{R_{0 i}^{3}}{R_{0 j}^{5}}+\frac{315}{256} \frac{R_{0 i}^{5}}{R_{0 j}^{4}}+\ldots ; \\
& b_{j, 1}=\frac{3}{8} \frac{R_{0 i}^{2}}{R_{0 j}^{4}}+\frac{15}{64} \frac{R_{0 i}^{4}}{R_{0 j}^{6}}+\frac{175}{1024} \frac{R_{0 i}^{6}}{R_{0 j}^{8}}+\ldots ; \quad b_{j, 2}=\frac{3}{2} \frac{R_{0 i}}{R_{0 j}^{3}}+\frac{5}{8} \frac{R_{0 i}^{3}}{R_{0 j}^{5}}+\frac{105}{256} \frac{R_{0 i}^{5}}{R_{0 j}^{7}}+\ldots
\end{aligned}
$$

\section{APPENDIX E: AMPLITUDES AND FREQUENCIES FOR SEP VIOLATION AND PLANETARY SIGNATURES}

\begin{tabular}{|c|c|c|c|c|c|c|c|c|c|c|c|c|}
\hline \multirow[b]{3}{*}{ Frequency } & \multicolumn{2}{|c|}{ Direct terms $\left(\mathcal{D}_{l}\right)$} & \multicolumn{10}{|c|}{ Indirect terms $\left(\mathcal{I}_{p q r}\right)$} \\
\hline & \multirow[b]{2}{*}{ Period (d) } & \multirow[b]{2}{*}{ Ampl. (m) } & \multicolumn{2}{|c|}{ Mars } & \multicolumn{2}{|c|}{ Jupiter } & \multicolumn{2}{|c|}{ Saturn } & \multicolumn{2}{|c|}{ Uranus } & \multicolumn{2}{|c|}{ Neptune } \\
\hline & & & $\overline{\text { Period (d) }}$ & Ampl. (m) & Period (d) & Ampl. (m) & $\overline{\text { Period (d) }}$ & Ampl. (m) & Period (d) & Ampl. (m) & Period (d) & Ampl. (m) \\
\hline$\overline{0}$ & $\infty$ & -22.99 & & & & & & & & & & \\
\hline$n_{1}-n_{3}$ & 115.9 & 1.51 & & & & & & & & & & \\
\hline$n_{j}-n_{3}$ & & & 747.35 & 0.36 & 398.80 & 232.53 & 378.09 & 47.28 & 369.66 & 4.91 & 367.49 & 4.59 \\
\hline$n_{1}-n_{j}$ & & & 100.32 & -0.30 & 89.79 & -134.53 & 88.69 & -26.71 & 88.22 & -2.75 & 88.10 & -2.56 \\
\hline$n_{j}+n_{1}-2 n_{3}$ & & & 137.14 & 0.29 & 163.34 & 121.87 & 167.09 & 24.03 & 168.79 & 2.47 & 169.24 & 2.29 \\
\hline $2 n_{1}-2 n_{3}$ & 57.9 & -0.58 & & & & & & & & & & \\
\hline $2 n_{1}+n_{j}-3 n_{3}$ & & & 62.81 & 0.06 & 67.79 & 27.58 & 68.42 & 5.47 & 68.71 & 0.56 & 68.78 & 0.52 \\
\hline $2 n_{1}-n_{j}-n_{3}$ & & & 53.77 & -0.02 & 50.59 & 20.72 & 50.24 & 4.81 & 50.09 & 0.53 & 50.05 & 0.50 \\
\hline $3 n_{1}-3 n_{3}$ & 38.6 & -0.19 & & & & & & & & & & \\
\hline $3 n_{1}+n_{j}-4 n_{3}$ & & & 40.73 & 0.02 & 42.77 & 7.99 & 43.02 & 1.58 & 43.13 & 0.16 & 43.16 & 0.15 \\
\hline $4 n_{1}+n_{j}-5 n_{3}$ & & & 30.14 & & 31.24 & 2.93 & 31.37 & 0.58 & 31.43 & 0.06 & 31.45 & 0.06 \\
\hline $3 n_{1}-n_{j}-2 n_{3}$ & & & 36.73 & & 35.22 & 2.12 & 35.05 & 0.56 & 34.97 & 0.06 & 34.95 & 0.06 \\
\hline $4 n_{1}-4 n_{3}$ & 29.0 & -0.11 & & & & & & & & & & \\
\hline $4 n_{1}-n_{j}-3 n_{3}$ & & & 27.89 & & 27.01 & 0.55 & 26.91 & 0.15 & 26.86 & 0.02 & 26.85 & 0.02 \\
\hline $5 n_{1}+n_{j}-6 n_{3}$ & & & 23.92 & & 24.61 & 0.49 & 24.69 & 0.09 & 24.73 & & 24.74 & \\
\hline $5 n_{1}-n_{j}-4 n_{3}$ & & & 22.48 & & 21.90 & -0.41 & 21.84 & -0.07 & 21.81 & & 21.80 & \\
\hline $6 n_{1}-n_{j}-5 n_{3}$ & & & 18.83 & & 18.42 & 0.26 & 18.37 & 0.05 & 18.35 & & 18.35 & \\
\hline $2 n_{j}-2 n_{3}$ & & & 373.67 & & 199.40 & & 189.04 & & 184.83 & & 183.74 & \\
\hline$n_{1}-2 n_{j}+n_{3}$ & & & 88.45 & & 73.29 & & 71.84 & & 71.22 & & 71.06 & \\
\hline$n_{1}+2 n_{j}-3 n_{3}$ & & & 167.96 & & 276.64 & & 299.40 & & 310.62 & & 313.73 & \\
\hline $5 n_{1}-5 n_{3}$ & 23.2 & 0.06 & & & & & & & & & & \\
\hline$\cdots$ & $\cdots$ & $\cdots$ & $\cdots$ & $\cdots$ & $\cdots$ & $\cdots$ & $\cdots$ & $\cdots$ & $\cdots$ & $\cdots$ & $\cdots$ & $\cdots$ \\
\hline
\end{tabular}

See Tables IV and V.

TABLE IV. SEP violation range signature: coefficients relative to the direct and indirect parts (for $\eta=1$ ). Only terms bigger than $1 \mathrm{~cm}$ are reported. See Eq. (32). 
TABLE V. Earth-Mercury range perturbation coefficients due to planetary effects. Only terms bigger than $10 \mathrm{~cm}$ are reported.

\begin{tabular}{|c|c|c|c|c|c|c|c|c|c|c|}
\hline \multirow[b]{3}{*}{ Frequency } & \multicolumn{10}{|c|}{ Coefficients $\mathcal{J}$ of Earth-Mercury range perturbation } \\
\hline & \multicolumn{2}{|c|}{ Mars } & \multicolumn{2}{|c|}{ Jupiter } & \multicolumn{2}{|c|}{ Saturn } & \multicolumn{2}{|c|}{ Uranus } & \multicolumn{2}{|c|}{ Neptune } \\
\hline & Period (d) & Ampl. (m) & Period (d) & Ampl. (m) & Period (d) & Ampl. (m) & Period (d) & Ampl. (m) & Period (d) & Ampl. (m) \\
\hline 0 & $\infty$ & -3630.05 & $\infty$ & -169686.9 & $\infty$ & -8031.6 & $\infty$ & -149.5 & $\infty$ & -45.7 \\
\hline $5 n_{j}-5 n_{3}$ & 149.5 & -3847.5 & 79.8 & -1244.3 & 75.6 & -8.6 & 73.9 & & 73.5 & \\
\hline $4 n_{j}-4 n_{3}$ & 186.8 & -10505.7 & 99.7 & -9969.9 & 94.5 & -124.9 & 92.4 & -0.5 & 91.9 & \\
\hline $3 n_{j}-3 n_{3}$ & 249.1 & -40416.7 & 132.9 & -94362.8 & 126.0 & -2140.4 & 123.2 & -18.7 & 122.5 & -3.6 \\
\hline $2 n_{j}-2 n_{3}$ & 373.7 & 1675569.7 & 199.4 & -1319529.2 & 189.0 & -53036.5 & 184.8 & -921.7 & 183.7 & -276.9 \\
\hline$n_{j}-n_{3}$ & 747.3 & 40176.8 & 398.8 & 2207243.3 & 378.1 & 135303.2 & 369.7 & 3503.0 & 367.5 & 1334.7 \\
\hline$n_{1}+5 n_{j}-6 n_{3}$ & 515.6 & -914.7 & 255.9 & -258.3 & 217.6 & -1.8 & 204.2 & & 201.0 & \\
\hline$n_{1}+4 n_{j}-5 n_{3}$ & 305.1 & -2735.8 & 714.2 & -2158.2 & 512.9 & -26.8 & 456.4 & -0.1 & 443.5 & \\
\hline$n_{1}+3 n_{j}-4 n_{3}$ & 216.7 & -12199.5 & 903.1 & -22017.2 & 1438.5 & -492.5 & 1944.8 & -4.3 & 2144.8 & -0.8 \\
\hline$n_{1}+2 n_{j}-3 n_{3}$ & 168.0 & 657422.1 & 276.6 & -359448.6 & 299.4 & -14122.6 & 310.6 & -243.1 & 313.7 & -72.9 \\
\hline$n_{1}+n_{j}-2 n_{3}$ & 137.1 & 29419.5 & 163.3 & 942637.0 & 167.1 & 55310.6 & 168.8 & 1405.8 & 169.2 & 533.1 \\
\hline$n_{1}-n_{3}$ & 115.9 & 112.1 & 115.9 & 6586.4 & 115.9 & 316.1 & 115.9 & 5.9 & 115.9 & 1.8 \\
\hline$n_{1}-n_{j}$ & 100.3 & -30212.5 & 89.8 & -990256.1 & 88.7 & -58250.5 & 88.2 & -1482.1 & 88.1 & -562.1 \\
\hline$n_{1}-2 n_{j}+n_{3}$ & 88.4 & -686619.7 & 73.3 & 412491.0 & 71.8 & 16445.2 & 71.2 & 285.0 & 71.1 & 85.6 \\
\hline$n_{1}-3 n_{j}+2 n_{3}$ & 79.1 & 12926.6 & 61.9 & 23846.6 & 60.4 & 534.4 & 59.7 & 4.7 & 59.5 & 0.9 \\
\hline$n_{1}-4 n_{j}+3 n_{3}$ & 71.5 & 2922.8 & 53.6 & 2337.1 & 52.1 & 29.03 & 51.4 & 0.1 & 51.2 & \\
\hline$n_{1}-5 n_{j}+4 n_{3}$ & 65.3 & 983.08 & 47.2 & 280.4 & 45.8 & 1.9 & 45.1 & & 45.0 & \\
\hline $2 n_{1}+5 n_{j}-7 n_{3}$ & 94.6 & -246.7 & 211.8 & -72.7 & 247.8 & -0.5 & 267.8 & & 273.7 & \\
\hline $2 n_{1}+4 n_{j}-6 n_{3}$ & 84.0 & -719.0 & 138.3 & -599.1 & 149.7 & -7.5 & 155.3 & & 156.9 & \\
\hline $2 n_{1}+3 n_{j}-5 n_{3}$ & 75.5 & -3083.9 & 102.7 & -5970.8 & 107.2 & -134.1 & 109.4 & -1.2 & 109.9 & -0.2 \\
\hline $2 n_{1}+2 n_{j}-4 n_{3}$ & 68.6 & 156661.1 & 81.7 & -93233.7 & 83.5 & -3685.2 & 84.4 & -63.6 & 84.6 & -19.1 \\
\hline $2 n_{1}+n_{j}-3 n_{3}$ & 62.8 & 6350.1 & 67.8 & 220937.0 & 68.4 & 13073.8 & 68.7 & 333.5 & 68.8 & 126.6 \\
\hline $2 n_{1}-2 n_{3}$ & 57.9 & -116.3 & 57.9 & -5171.2 & 57.9 & -243.9 & 57.9 & -4.5 & 57.9 & -1.4 \\
\hline $2 n_{1}-n_{j}-n_{3}$ & 53.8 & -4587.1 & 50.6 & -104942.3 & 50.2 & -5864.9 & 50.1 & -145.9 & 50.0 & -55.0 \\
\hline $2 n_{1}-2 n_{j}$ & 50.2 & -98799.3 & 44.9 & 43063.2 & 44.3 & 1638.8 & 44.1 & 27.8 & 44.0 & 8.3 \\
\hline $2 n_{1}-3 n_{j}+n_{3}$ & 47.0 & 1730.3 & 40.4 & 3452.4 & 39.7 & 79.2 & 39.4 & 0.7 & 39.3 & 0.1 \\
\hline $2 n_{1}-4 n_{j}+2 n_{3}$ & 44.2 & 356.7 & 36.6 & 259.9 & 35.9 & 3.2 & 35.6 & & 35.5 & \\
\hline $2 n_{1}-5 n_{j}+3 n_{3}$ & 41.8 & 113.7 & 33.6 & 29.7 & 32.8 & 0.2 & 32.5 & & 32.4 & \\
\hline $3 n_{1}+5 n_{j}-8 n_{3}$ & 52.1 & -73.4 & 74.9 & -21.7 & 79.0 & -0.1 & 80.9 & & 81.4 & \\
\hline $3 n_{1}+4 n_{j}-7 n_{3}$ & 48.7 & -213.3 & 63.0 & -178.5 & 65.3 & -2.2 & 66.4 & & 66.6 & \\
\hline $3 n_{1}+3 n_{j}-6 n_{3}$ & 45.7 & -912.1 & 54.4 & -1777.2 & 55.7 & -39.9 & 56.3 & -0.3 & 56.4 & \\
\hline $3 n_{1}+2 n_{j}-5 n_{3}$ & 43.1 & 46092.3 & 47.9 & -27592.5 & 48.5 & -1090.9 & 48.8 & -18.8 & 48.9 & -5.6 \\
\hline $3 n_{1}+n_{j}-4 n_{3}$ & 40.7 & 1850.0 & 42.8 & 64817.3 & 43.0 & 3838.1 & 43.1 & 97.9 & 43.2 & 37.2 \\
\hline $3 n_{1}-3 n_{3}$ & 38.6 & -37.9 & 38.6 & -1694.5 & 38.6 & -80.0 & 38.6 & -1.5 & 38.6 & -0.5 \\
\hline $3 n_{1}-n_{j}-2 n_{3}$ & 36.7 & -1330.5 & 35.2 & -32709.3 & 35.0 & -1851.5 & 35.0 & -46.3 & 35.0 & -17.5 \\
\hline $3 n_{1}-2 n_{j}-n_{3}$ & 35.0 & -27437.1 & 32.4 & 7858.8 & 32.1 & 268.9 & 31.9 & 4.3 & 31.9 & 1.3 \\
\hline $3 n_{1}-3 n_{j}$ & 33.4 & 451.4 & 29.9 & 581.6 & 29.6 & 12.4 & 29.4 & 0.1 & 29.4 & \\
\hline $3 n_{1}-4 n_{j}+n_{3}$ & 32.0 & 101.3 & 27.8 & 96.5 & 27.4 & 1.2 & 27.2 & & 27.2 & \\
\hline $3 n_{1}-5 n_{j}+2 n_{3}$ & 30.7 & 30.4 & 26.0 & 8.0 & 25.6 & & 25.4 & & 25.3 & \\
\hline $4 n_{1}+5 n_{j}-9 n_{3}$ & 35.9 & -28.1 & 45.5 & -8.4 & 47.0 & & 47.6 & & 47.8 & \\
\hline $4 n_{1}+4 n_{j}-8 n_{3}$ & 34.3 & -81.0 & 40.8 & -68.8 & 41.8 & -0.9 & 42.2 & & 42.3 & \\
\hline $4 n_{1}+3 n_{j}-7 n_{3}$ & 32.8 & -342.7 & 37.0 & -680.9 & 37.6 & -15.3 & 37.9 & -0.1 & 37.9 & \\
\hline $4 n_{1}+2 n_{j}-6 n_{3}$ & 31.4 & 17008.4 & 33.9 & -10513.7 & 34.2 & -416.9 & 34.4 & -7.2 & 34.4 & -2.2 \\
\hline $4 n_{1}+n_{j}-5 n_{3}$ & 30.1 & 660.3 & 31.2 & 23828.2 & 31.4 & 1415.0 & 31.4 & 36.1 & 31.4 & 13.7 \\
\hline $4 n_{1}-4 n_{3}$ & 29.0 & -19.1 & 29.0 & -873.7 & 29.0 & -41.3 & 29.0 & -0.8 & 29.0 & -0.2 \\
\hline $4 n_{1}-n_{j}-3 n_{3}$ & 27.9 & -419.8 & 27.0 & -9569.0 & 26.9 & -535.5 & 26.9 & -13.3 & 26.9 & -5.0 \\
\hline $4 n_{1}-2 n_{j}-2 n_{3}$ & 26.9 & -7859.3 & 25.3 & 2266.9 & 25.1 & 79.4 & 25.0 & 1.3 & 25.0 & 0.4 \\
\hline $4 n_{1}-3 n_{j}-n_{3}$ & 26.0 & 115.9 & 23.8 & 80.1 & 23.6 & 1.5 & 23.5 & & 23.4 & \\
\hline $4 n_{1}-4 n_{j}$ & 25.1 & 22.4 & 22.4 & 8.5 & 22.2 & & 22.1 & & 22.0 & \\
\hline
\end{tabular}


Coefficients $\mathcal{J}$ of Earth-Mercury range perturbation

\begin{tabular}{|c|c|c|c|c|c|c|c|c|c|c|}
\hline \multirow[b]{2}{*}{ Frequency } & \multicolumn{2}{|c|}{ Mars } & \multicolumn{2}{|c|}{ Jupiter } & \multicolumn{2}{|c|}{ Saturn } & \multicolumn{2}{|c|}{ Uranus } & \multicolumn{2}{|c|}{ Neptune } \\
\hline & Period (d) & Ampl. (m) & Period (d) & Ampl. (m) & Period (d) & $\overline{\text { Ampl. (m) }}$ & Period (d) & Ampl. (m) & Period (d) & $\overline{\text { Ampl. (m) }}$ \\
\hline $4 n_{1}-5 n_{j}+n_{3}$ & 24.3 & 7.9 & 21.3 & 3.0 & 20.9 & & 20.8 & & 20.8 & \\
\hline $5 n_{1}+5 n_{j}-10 n_{3}$ & 27.4 & -1.2 & 32.7 & -0.1 & 33.4 & & 33.8 & & 33.8 & \\
\hline $5 n_{1}+4 n_{j}-9 n_{3}$ & 26.5 & -4.7 & 30.2 & -1.7 & 30.7 & & 30.9 & & 31.0 & \\
\hline $5 n_{1}+3 n_{j}-8 n_{3}$ & 25.6 & -28.7 & 28.1 & -26.5 & 28.4 & -0.6 & 28.5 & & 28.6 & \\
\hline $5 n_{1}+2 n_{j}-7 n_{3}$ & 24.7 & 2137.1 & 26.2 & -695.7 & 26.4 & -26.0 & 26.5 & -0.4 & 26.5 & -0.1 \\
\hline $5 n_{1}+n_{j}-6 n_{3}$ & 23.9 & 136.7 & 24.6 & 3295.1 & 24.7 & 186.5 & 24.7 & 4.7 & 24.7 & 1.8 \\
\hline $5 n_{1}-5 n_{3}$ & 23.2 & 9.3 & 23.2 & 440.9 & 23.2 & 20.9 & 23.2 & 0.4 & 23.2 & 0.1 \\
\hline $5 n_{1}-n_{j}-4 n_{3}$ & 22.5 & -229.9 & 21.9 & -8103.3 & 21.8 & -479.6 & 21.8 & -12.2 & 21.8 & -4.6 \\
\hline $5 n_{1}-2 n_{j}-3 n_{3}$ & 21.8 & -6284.5 & 20.8 & 3697.7 & 20.6 & 145.1 & 20.6 & 2.5 & 20.6 & 0.7 \\
\hline $5 n_{1}-3 n_{j}-2 n_{3}$ & 21.2 & 127.5 & 19.7 & 242.9 & 19.6 & 5.4 & 19.5 & & 19.5 & \\
\hline $5 n_{1}-4 n_{j}-n_{3}$ & 20.6 & 30.2 & 18.8 & 24.0 & 18.6 & 0.3 & 18.5 & & 18.5 & \\
\hline $5 n_{1}-5 n_{j}$ & 20.1 & 10.5 & 18.0 & 2.9 & 17.7 & & 17.6 & & 17.6 & \\
\hline $6 n_{1}-n_{j}-5 n_{3}$ & 18.8 & 2.4 & 18.4 & 253.3 & 18.4 & 16.2 & 18.4 & 0.4 & 18.3 & 0.2 \\
\hline $6 n_{1}-2 n_{j}-4 n_{3}$ & 18.4 & -2.9 & 17.6 & -142.9 & 17.5 & -6.6 & 17.5 & -0.1 & 17.5 & \\
\hline $6 n_{1}-3 n_{j}-3 n_{3}$ & 17.9 & -0.3 & 16.9 & -4.5 & 16.7 & -0.1 & 16.7 & & 16.7 & \\
\hline $6 n_{1}-4 n_{j}-2 n_{3}$ & 17.5 & -0.1 & 16.2 & -0.5 & 16.0 & & 16.0 & & 16.0 & \\
\hline $6 n_{1}-5 n_{j}-n_{3}$ & 17.1 & & 15.5 & & 15.4 & & 15.3 & & 15.3 & \\
\hline $7 n_{1}-2 n_{j}-5 n_{3}$ & 15.9 & -0.6 & 15.3 & -28.8 & 15.2 & -1.3 & 15.2 & & 15.2 & \\
\hline $7 n_{1}-3 n_{j}-4 n_{3}$ & 15.5 & -0.2 & 14.7 & -3.0 & 14.6 & & 14.6 & & 14.6 & \\
\hline $7 n_{1}-4 n_{j}-3 n_{3}$ & 15.2 & & 14.2 & -0.1 & 14.1 & & 14.0 & & 14.0 & \\
\hline $7 n_{1}-5 n_{j}-2 n_{3}$ & 14.9 & & 13.7 & & 13.6 & & 13.5 & & 13.5 & \\
\hline $8 n_{1}-3 n_{j}-5 n_{3}$ & 13.7 & & 13.1 & -0.4 & 13.0 & & 13.0 & & 13.0 & \\
\hline $8 n_{1}-4 n_{j}-4 n_{3}$ & 13.4 & & 12.6 & -0.1 & 12.6 & & 12.5 & & 12.5 & \\
\hline $8 n_{1}-5 n_{j}-3 n_{3}$ & 13.2 & & 12.3 & & 12.2 & & 12.1 & & 12.1 & \\
\hline$\cdots$ & $\cdots$ & $\cdots$ & $\cdots$ & . & $\cdots$ & $\cdots$ & $\cdots$ & . & $\cdots$ & . \\
\hline
\end{tabular}

[1] L. Iess and G. Boscagli, Advanced radio science instrumentation for the mission BepiColombo to Mercury, Planet. Space Sci. 49, 1597 (2001).

[2] A. Milani, D. Vokrouhlický, D. Villani, C. Bonanno, and A. Rossi, Testing general relativity with the BepiColombo radio science experiment, Phys. Rev. D 66, 082001 (2002).

[3] T. Damour and D. Vokrouhlický, Equivalence principle and the Moon, Phys. Rev. D 53, 4177 (1996).

[4] G. Congedo and F. De Marchi, Testing the strong equivalence principle with spacecraft ranging towards the nearby Lagrangian points, Phys. Rev. D 93, 102003 (2016).

[5] J. G. Williams, S. G. Turyshev, and D. H. Boggs, Lunar Laser Ranging tests of the equivalence principle with the Earth and Moon, Int. J. Mod. Phys. D18, 1129 (2009).

[6] K. Nordtvedt, Testing Relativity with Laser Ranging to the Moon, Phys. Rev. 170, 1186 (1968).

[7] E. G. Adelberger, J. H. Gundlach, B. R. Heckel, S. Hoedl, and S. Schlamminger, Torsion balance experiments: a low-energy frontier of particle physics, Prog. Part. Nucl. Phys. 62, 102 (2009).

[8] P. Touboul, G. Métris, V. Lebat, and A. Robert, The MICROSCOPE experiment, ready for the in-orbit test of the equivalence principle, Classical Quantum Gravity 29, 184010 (2012)

[9] T. W. Murphy, Jr., E. G. Adelberger, J. B. R. Battat, C. D. Hoyle, N. H. Johnson, R. J. McMillan, C. W. Stubbs, and H. E. Swanson, APOLLO: millimeter lunar laser ranging, Classical Quantum Gravity 29, 184005 (2012).

[10] S. G. Turyshev and J. G. Williams, Space based tests of gravity with laser ranging, Int. J. Mod. Phys. D16, 2165 (2007).

[11] S. G. Turyshev, J. G. Williams, M. Shao, J. D. Anderson, K. L. Nordtvedt, Jr., and T. W. Murphy, Jr., Laser ranging to the Moon, Mars and Beyond, arXiv:gr-qc/0411082.

[12] S. G. Turyshev, W. Farr, W. M. Folkner, A. R. Girerd, H. Hemmati, T. W. Murphy, J. G. Williams, and J. J. Degnan, 
Advancing tests of relativistic gravity via laser ranging to Phobos, Exp. Astron. 28, 209 (2010).

[13] A. Milani, G. Tommei, D. Vokrouhlický, E. Latorre, and S. Cicalò, Relativistic models for the BepiColombo Radioscience experiment, in Relativity in Fundamental Astronomy: Dynamics, Reference Frames, and Data Analysis, IAU Symposium Vol. 261, edited by S. A. Klioner, P. K. Seidelmann, and M. H. Soffel (Cambridge University Press, 2010), pp. 356-365.

[14] S. Cicalò, G. Schettino, S. Di Ruzza, E. M. Alessi, G. Tommei, and A. Milani, The BepiColombo MORE gravimetry and rotation experiments with the ORBIT14 software, Mon. Not. R. Astron. Soc. 457, 1507 (2016).

[15] G. Tommei, L. Dimare, D. Serra, and A. Milani, On the Juno radio science experiment: models, algorithms and sensitivity analysis, Mon. Not. R. Astron. Soc. 446, 3089 (2015).

[16] G. Tommei, A. Milani, and D. Vokrouhlický, Light-time computations for the BepiColombo Radioscience Experiment, Celest. Mech. Dyn. Astron. 107, 285 (2010).

[17] A. Milani and G. Gronchi, Theory of Orbit Determination (Cambridge University Press, Cambridge, England, 2010).

[18] Every single arc has its own set of initial conditions; in this way, the actual errors in the orbit propagation, due to lack of knowledge in the nongravitational dynamical model, can be reduced by an overparametrization of the initial conditions.

[19] E. M. Alessi, S. Cicalò, A. Milani, and G. Tommei, Desaturation manoeuvres and precise orbit determination for the BepiColombo mission, Mon. Not. R. Astron. Soc. 423, 2270 (2012).

[20] G. Schettino, S. Cicalò, S. Di Ruzza, and G. Tommei, The relativity experiment of MORE: Global full-cycle simulation and results, in International Workshop in Metrology for Aerospace (IEEE Proceedings, New York, 2015), pp. 141-145.

[21] K. Nordtvedt, Jr., Post-Newtonian metric for a general class of scalar-tensor gravitational theories and observational consequences, Astrophys. J. 161, 1059 (1970).

[22] Since, from a relativistic point of view, we are not dealing with a generic space-time but with the one where we are "now," we need to solve for the initial conditions of the bodies which affect the measurements, i.e., Mercury and EMB. The other planetary ephemerides are taken by Jet Propulsion Laboratory (JPL) ones.

[23] G. Tommei, On the BepiColombo and Juno radio science experiments: Precise models and critical estimates, in On the BepiColombo and Juno Radioscience Experiments: Precise
Models and Critical Estimates, Metrology for Aerospace (MetroAeroSpace), 2015 (IEEE, Benevento, Italy, 2015), pp. 323-328.

[24] B. Luzum, N. Capitaine, A. Fienga, W. Folkner, T. Fukushima, J. Hilton, C. Hohenkerk, G. Krasinsky, G. Petit, E. Pitjeva, M. Soffel, and P. Wallace, The IAU 2009 system of astronomical constants: the report of the IAU working group on numerical standards for Fundamental Astronomy, Celest. Mech. Dyn. Astron. 110, 293 (2011).

[25] T. D. Moyer, Formulation for Observed and Computed Values of Deep Space Network Data Types for Navigation (Wiley, Hoboken, New Jersey, 2003).

[26] N. Ashby, P. L. Bender, and J. M. Wahr, Future gravitational physics tests from ranging to the BepiColombo Mercury planetary orbiter, Phys. Rev. D 75, 022001 (2007).

[27] W. H. Clohessy and R. S. Wiltshire, Terminal guidance for satellite rendezvous, J. Aerosp. Sci. 27, 653 (1960).

[28] J. D. Anderson, M. Gross, K. L. Nordtvedt, and S. G. Turyshev, The solar test of the equivalence principle, Astrophys. J. 459, 365 (1996).

[29] D. Serra, Satellite geodesy on other planets, Ph.D. thesis, University of Pisa, 2016.

[30] S. A. Stern, F. Bagenal, K. Ennico, G. R. Gladstone, W. M. Grundy, W. B. McKinnon, J. M. Moore, C. B. Olkin, J. R. Spencer, H. A. Weaver, L. A. Young et al., The Pluto system: Initial results from its exploration by New Horizons, Science 350 (2015).

[31] R. S. Park, A. S. Konopliv, B. Bills, J. Castillo-Rogez, S. W. Asmar, N. Rambaux, C. A. Raymond, C. T. Russell, M. T. Zuber, A. Ermakov, S. D. King, and M. D. Rayman, Gravity science investigation of Ceres from Dawn, in Proceedings of 47th Lunar and Planetary Science Conference, The Woodlands, Texas, 2016 (2016), Vol. 47, p. 1781.

[32] S. Mouret, J. L. Simon, F. Mignard, and D. Hestroffer, The list of asteroids perturbing the Mars orbit to be seen during future space missions, Astron. Astrophys. 508, 479 (2009).

[33] A. S. Konopliv, S. W. Asmar, R. S. Park, B. G. Bills, F. Centinello, A. B. Chamberlin, A. Ermakov, R. W. Gaskell, N. Rambaux, C. A. Raymond, C. T. Russell, D. E. Smith, P. Tricarico, and M. T. Zuber, The Vesta gravity field, spin pole and rotation period, landmark positions, and ephemeris from the Dawn tracking and optical data, Icarus 240, 103 (2014).

[34] S. Schweighart and R. Sedwick, A perturbative analysis of geopotential disturbances for satellite cluster formation flying, in Aerospace Conference (IEEE Proceedings, New York, 2001), Vol. 2, pp. 1001-1019. 